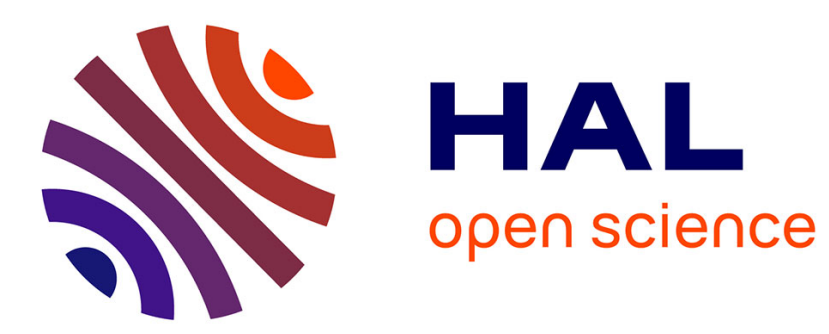

\title{
High order harmonic balance formulation of free and encapsulated microbubbles
}

Marie-Christine Pauzin, Serge Mensah, Bruno Cochelin, Jean-Pierre Lefebvre

\section{To cite this version:}

Marie-Christine Pauzin, Serge Mensah, Bruno Cochelin, Jean-Pierre Lefebvre. High order harmonic balance formulation of free and encapsulated microbubbles. Journal of Sound and Vibration, 2011, 330 (5), pp.987-1004. 10.1016/j.jsv.2010.09.014 . hal-00430105

\section{HAL Id: hal-00430105 https://hal.science/hal-00430105}

Submitted on 5 Nov 2009

HAL is a multi-disciplinary open access archive for the deposit and dissemination of scientific research documents, whether they are published or not. The documents may come from teaching and research institutions in France or abroad, or from public or private research centers.
L'archive ouverte pluridisciplinaire HAL, est destinée au dépôt et à la diffusion de documents scientifiques de niveau recherche, publiés ou non, émanant des établissements d'enseignement et de recherche français ou étrangers, des laboratoires publics ou privés. 


\title{
High order harmonic balance formulation of free and encapsulated microbubbles
}

\author{
Marie-Christine Pauzin, Serge Mensah*, Bruno Cochelin, Jean-Pierre Lefebvre \\ CNRS - Laboratoire de Mécanique et d'Acoustique; \\ 31 chemin Joseph Aiguier, 13402 Marseille, Cedex 20, France
}

\begin{abstract}
The radial responses of free or encapsulated micro-bubbles excited by a plane wave of large wavelength are governed by NonLinear Ordinary Differential Equations (NL-ODEs). The nonlinear frequency response details the harmonic content of the time response and constitutes the expected outcome of a high order harmonic analysis. In this paper, a high order harmonic balance analysis of the "RNNP" (bubble), Hoff and Marmottant (contrast agents) models are performed with the open-source Manlab ${ }^{\circledR}$ software extensively described in a previous paper. With this purpose, the original NL-ODEs are recast into nonlinear systems for which the non-linearities are at most quadratic. In the spectral domain, this recast provides close form and aliasing-free solutions of arbitrary large number of harmonics. Relevant quantities such as primary and secondary resonances or the nonlinear amplitude threshold of the excitation wave are evaluated. The frequency curves drawn characterize the bending and quantify the jump frequencies and amplitudes of each harmonic component. The results obtained with this predictive methodology confirm the significant aid it may provide in the derivation of bubble nonlinear detection and sizing techniques or in the design of contrast agents and their optimal ultrasound activation.
\end{abstract}

Keywords: ultrasound, diffraction tomography, near-field

PACS: 43.25.Yw 43.25.Ts 43.25.Zx

\section{Introduction}

The study of the nonlinear response of microbubbles to ultrasound excitations is of interest for the detection and sizing of bubbles $[1,2,3,4]$ in the engineering field, from propeller damage induced by cavitation to drag reduction by supercavitation $[5,6]$, valve leakage, chemical process control [7], monitoring of liquid sodium coolant for nuclear reactors [8,9], and in the biomedical field for decompression sickness prevention (in diving and space extravehicular activities

\footnotetext{
*Corresponding author.

Email address: mensah@lma.cnrs-mrs.fr (Serge Mensah)
} 
[10]) and medical imaging with contrast agents $[13,15,16,17,18,19,20]($ i.e. encapsulated microbubbles). For the latter field of activity, in specific conditions, it has been demonstrated that the sole second harmonic signal generated by bubbles and Ultrasonic Contrast Agents (UCA) may provide enhanced contrast information (bubble to tissue discrimination) if compared with the global signal content also comprising the fundamental component. This possibility of bubble/tissue discrimination while working on the second harmonic is founded on the fact that nonlinear response of tissues is drastically weaker than that of bubbles or contrast agents. The second harmonic imaging potential capability of UCA has been deeply investigated by a number of research institutions and private companies in order to derive relevant physical parameters improving the second harmonic backscattering cross section. However, in practical use, second harmonic imaging suffers from several drawbacks: firstly, it is altered by an attenuation of the second harmonic components higher than that undergone by the fundamental one, reducing implicitly the range (depth of penetration) of 2nd harmonic imaging. In addition, the significant second harmonic signal produced by the tissue itself impinges on the contrast of the bubbles or agents[21, 22]. These limitations could be partly overcome if it were possible to exploit most of the major harmonics, ultraharmonics and subharmonics generated by the pulsating bubbles. On the other hand, one can expect to improve not only range and resolution by using specific coded sequences (pulse compression techniques) but also the bubble or contrast agent signature characterization by a fine analysis, for instance a time-frequency analysis, of the global response (including the fundamental). The importance of the large bubble oscillations at specific frequencies, the so-called resonance frequencies, motivates the search for the characterization of the nonlinear acoustical response of the bubble according to the local context. This will pave the way for the design of more or less accurate inversion procedures able to give information related to, for instance, the bubble size, the possible attachment of the targeted contrast agent to neighboring tissues, the mechanical behavior of these neighboring tissues. Thus, in order to render the tissue/bubble separation more acute or to improve the detection and characterization capabilities, we present nonlinear frequency responses of bubbles and contrast agents obtained by using various models and a high order Harmonic Balance Method (HBM) combined with a Continuation Method. The method, implemented in a useful software[23], has been extensively described in a previous paper [24]. It generates high precision approximations of the full responses, including stable and unstable solutions, the degree of precision depending on the number, which may be high, of harmonic terms of the driving solicitation taken into account.

Since the main features of the non-linear as well as of the linear acoustic responses of microbubbles to ultrasound lie in their radial oscillations, this paper, like most of the literature on the subject, is devoted to the radial oscillations of spherical bare or coated microbubbles. For bare bubbles, the modeling of the problem leads to the so-called Rayleigh-Plesset equation and its generalization, the RPNNP model (referring to Rayleigh, Plesset, Nolting, Neppiras and Poritsky; terminology suggested by Lauterborn) [25], or to its alternative 
within the Keller-Miksis model [26]. For coated bubbles, modeling leads to equivalent equations that differentiate themselves from considerations on the shell behavior (viscosity, hyperelasticity ...). Among the various models of the agents we can quote those of Church [13], Hoff [15] and Marmottant [17]. The corresponding NonLinear Ordinary Differential Equations (NL-ODE) thus derived drive the time evolution of the radius of the bubble in response to the ultrasonic solicitation. In most cases a harmonic activation produced by an incident harmonic ultrasonic plane wave is considered. When the amplitudes of the oscillations are not too important, classical perturbation methods[28, 29] may apply and have been applied to solve these NL-ODE, resulting in a small parameter dependency and control. In that category, one may classify all analytical papers based on low order (2nd or 3rd) approximations, in particular the historical (and inexplicably rarely cited) paper of Nayfeh [27] who used a multiple scales method that allowed him to draw frequency responses exhibiting the natural "softening" behavior of the bubbles. The simplicity of the parametrization generates a great interest in this method which was used by several authors $[30,31]$, whereas Prosperetti [32] preferred to employ another classical perturbation method, the method of Averaging. With the same purpose of drawing frequency responses, developments to second order small parameter combined with second order harmonic balance were chosen by many authors in order to find explicit formulations, for example Church [13] and Khismatullin [33]. Following Lauterborn[25], other authors chose the opposite way of a full numerical resolution in the time-domain, followed by a Fourier Analysis in order to extract the transfer function: $[26,34]$ and more recently [35, 16, 19, 18, 20]. The method of purely numerical resolution of the time-domain ODE is currently the unique one leading to a high precision approximation of the solution. But, only the stable solutions of the equations can be found and exploration of hysteretic solutions and of each harmonic and sub-harmonic component of the response are not easy tasks. A Harmonic Balance method, without any small-parameter development, is more suitable for the objective of high accuracy frequency response assessment. However, when oscillations are large, i.e. for resonances and near them, a development with a high number of harmonics is required if sufficient precision is expected. Since the explicit balancing on a large (greater than or equal to two) number of harmonics is rather cumbersome, an automated balancing technique has been employed here. The technique, combining a Harmonic Balance Method (HBM) and a Continuation Method within the framewok of Asymptotic Numerical Methods (ANM) [36], is detailed and illustrated with various applications in the paper [24]. It resulted in an interactive free and open access software written for a Matlab ${ }^{\circledR}$ environment and named Manlab ${ }^{\circledR}$ [23]. This software is intensely used in this paper for three models of microbubbles: the RPNNP model [25] which reproduces a free microbubbles response and two other models that describe thinly encapsulated microbubbles. Among the latter, the first one, owed to Hoff [15], is a thin-shelled version of the Church model [13], the second one due to Marmottant [17] accounts for buckling and rupture. The last one is however limited here in the elastic behavior domain of the shell. These two models are recognized to be well suited to the last 
generation of UCA, the polymer-shelled contrast agents and the lipid-shelled contrast agents respectively.

In the present study, for numerical experiment, it is assumed that the radial non linear behavior of the micro-bubble or the contrast agent is sufficiently characterized through its first 3 components (the fundamental plus two harmonics). With this aim in view, the expansion of the solutions is indeed performed on the first four harmonics to which we add the null frequency component. In practice, this should be sufficient when the attenuation coefficient of the host medium is, for instance, linearly dependent on the location depth of the bubble and on the frequency which is the case for "soft" tissues in biomedical engineering. Free bubbles in water may require higher Fourier decomposition to be modeled with arbitrary accuracy because of the low water viscosity that reduces the importance of the absorption phenomenon. The dedicated software used does not present any difficulty: it is founded on a simple principle, the quadratic recast, that enables its developers to avoid any iterative process (no problem of convergence and no unpredictable computation cost), any temporal discretization (it is a frequency domain approach), any use of numerical scheme in the computation of the derivative (the jacobian is calculated analytically and easily). Thus, the original bubble models are transformed into new non-linear systems (extended state equations) for which the non-linearities are at most quadratic. The application of the harmonic balance method on quadratic models is recognized at once even if one searches for large harmonic number solutions.

In order to give an explicit view of the transformation procedure, we start by presenting the procedure used to recast the nonlinear system in order to get at most quadratic polynomial nonlinearities. With this purpose, we consider the simple case of the forced Duffing oscillator [24]. Indeed, this simple model is quite representative of the free bubble radial oscillations in a viscous fluid as described by Lauterborn.

\section{Generic model: Forced Duffing oscillator.}

The normalized forced Duffing oscillator is described by the non-autonomous equation:

$$
\ddot{u}+2 \mu \dot{u}+u+\alpha u^{3}=f \cos (\lambda t) .
$$

In this model, the damping coefficient $\mu$, the degree of nonlinearity $\alpha$ and the force amplitude $f$ are constant; the forcing angular frequency is treated as the varying parameter $\lambda$. By using $v(t)=\dot{u}(t)$ and $w(t)=u^{2}(t)$, this equation can be recast in a quadratic form:

$$
\left\{\begin{aligned}
\dot{u} & = \\
\dot{v} & =f \cos (\lambda t) \\
\underbrace{}_{m(\dot{Z})} & =\underbrace{0}_{c(t, \lambda)} \underbrace{-2 \mu v-u}_{l(Z)} \begin{array}{l}
v \\
w \\
\begin{array}{c}
-\alpha u w \\
-u u
\end{array}
\end{array}
\end{aligned}\right.
$$

where $Z=[u, v, w]^{t}$, and the forcing term is deliberately put into $c$. The forcing angular frequency is related to the response frequency by putting $\omega=p \lambda$ ( $p$ is 
an integer). In the Harmonic Balance Method, the term $u(t)$ is expanded into harmonics with respect to $\omega$. Thus the compact expression of the equivalent quadratic system is:

$$
m(\dot{Z})=c+l(Z)+q(Z, Z),
$$

The unknown vector $Z$ (size $N_{e}$ ) contains the original components $u$ and $v$ plus the auxiliary variable $w$ that has been added to transform (1) into the quadratic form (2). The right hand size of (2) comprises vector $c$ that is independent of $Z ; m$ and $l$ are linear operators acting on $Z ; q$ is a quadratic vector operator that is linear with respect to both entries.

The harmonic balance method consists in searching periodic solutions to Eq. (2), these solutions being expressed as truncated Fourier series

$$
Z(t) \approx Z_{0}+\sum_{k=1}^{H}\left(a_{k} \cos k \lambda t+b_{k} \sin k \lambda t\right) .
$$

The substitution of this expression in system (2) and the balance of the terms corresponding to the different frequency components $\left(\left(a_{k}, b_{k}\right), \quad 1 \leqslant k \leqslant H\right)$ leads to a system of $(2 H+1) \times N_{e}$ algebraic equations for $(2 H+1) \times N_{e}+1$ unknowns: the $(2 H+1) \times N_{e}$ components of vector $Z$ and angular frequency $\lambda$. The branches of solutions of this algebraic system are then followed by a dedicated continuation technique (ANM) by fixing the path parameter $\lambda$. This procedure provides only approximate solutions since in expansion (4), all the harmonic terms greater than $H$ are not considered. However, if the number of harmonics $H$ is large enough, accurate solutions can be expected.

Figures (1) and (2) show the frequency-amplitude diagrams of the responses obtained in the case of a "hardening" system $(\alpha \geq 0)$. A classical bent resonance curve can be observed, as well as some additional peaks corresponding to superharmonic resonances. Note that only the individual amplitudes $A_{i}=\sqrt{a_{i}^{2}+b_{i}^{2}}$ of the odd harmonics have been plotted, since the even harmonics $\left(A_{0}, A_{2}\right.$, $\left.A_{4}, \ldots\right)$ are zero. In this example, the computation of this branch required 25 steps of MAN-continuation when 5 harmonics were included, and 35 steps when 9 harmonics were included. The curves obtained for harmonics 1 and 3 were slightly hanged when shifting from $H=5$ (solid line) to $H=9$ (broken line) harmonics in Eq. (4). The curve obtained for $A_{5}$ is more affected, which confirms the logical result according to which more than 5 harmonics have to be used to obtain accurate results with harmonic 5 . We also established that the curve obtained for $A_{5}$ was only very slightly modified when shifting from $H=9$ to $H=11$ harmonics in Eq. (4). The frequency responses of "softening" systems $(\alpha \leq 0)$ are shown in figures (3) and (4) which reveal instabilities and the so-called jump-frequencies. Latter, we shall see that bubbles and contrast agents are mainly softening systems. At the jump-down (respectively the jumpup) frequency, the vibration amplitude of the system suddenly jumps-down (paths [3]-[4] on the curves)(resp. jumps-up (paths [3']-[2])) when it is excited harmonically with slowly changing frequency. The frequencies at which these 


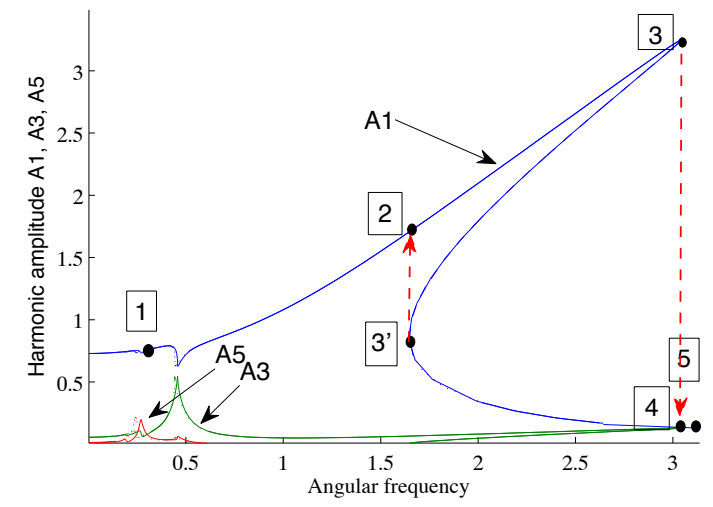

Figure 1: Duffing oscillator, Eq. (1) with $\mu=0.05, f=1.0$ and $\alpha=1$, "hardening" system, as in ([39]). The figure shows the amplitude of harmonics 1,3 and 5 versus forcing angular frequency $\lambda$. Continuous line : results when 5 harmonics are included $(\mathrm{H}=5$ in Eq. (4)). Dotted line : results when 9 harmonics are included.

jumps occur depend upon whether the modulation is increasing or decreasing and whether the nonlinearity is hardening (Fig. 1) or softening (Fig. 3)

Besides, when assuming a harmonic balance restricted to the fundamental component, the jump-down frequency of the Duffing oscillator is written [37]

$$
\omega_{J} \approx \frac{1}{2^{\frac{1}{2}}}\left(1+\left[1+\frac{3 \alpha}{4 \mu^{2}}\right]^{\frac{1}{2}}\right)^{\frac{1}{2}} .
$$

and the amplitude of vibration at this frequency is given by

$$
A_{J} \approx\left(\frac{2}{3 \alpha}\left(\left[1+\frac{3 \alpha}{4 \mu^{2}}\right]^{\frac{1}{2}}-1\right)\right)^{\frac{1}{2}}
$$

For $\omega_{J}$ to be real for a softening system, the following condition is necessary:

$$
\alpha \geq \alpha_{J}=-4 \mu^{2} / 3 .
$$

If this condition is not met, then a jump-down does not occur.

Furthermore, if the assumption $3 \alpha /\left(4 \mu^{2}\right) \ll 1$ is valid (this is quite restrictive if the damping is light), the jump-down frequency is written $\omega_{J} \approx 1+3 \alpha / 32 \mu^{2}$ and the amplitude at the jump-down frequency is given by $A_{J} \approx 1 / 2 \mu$. Then, the degree of nonlinearity alone is not the important parameter but how it compares with the damping, i.e. the ratio $3 \alpha / 4 \mu^{2}$ is the relevant parameter.

Thus, in first order approximation, the amplitude of the system response is lead principally by the damping, the deviation of the jump-down frequency from the natural frequency of the associated linear system (measurements obtained with an excitation force of low level) is dependent of the degree of nonlinearity and 


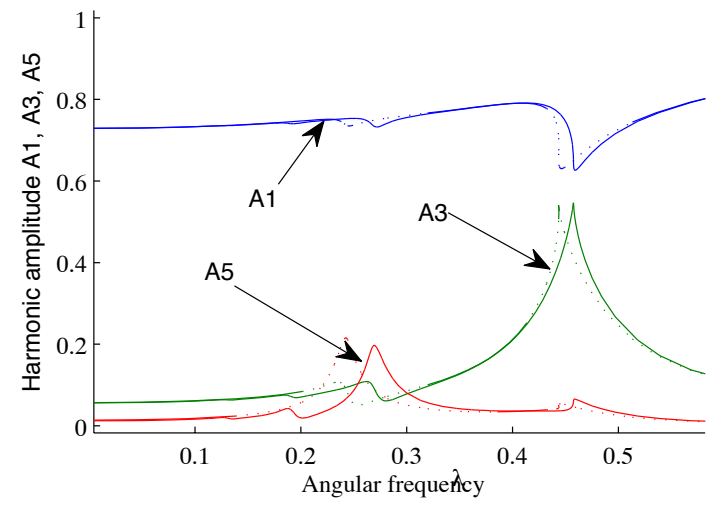

Figure 2: Duffing oscillator, same conditions as in Fig. (1): zoom of the region $\omega<0.6$.

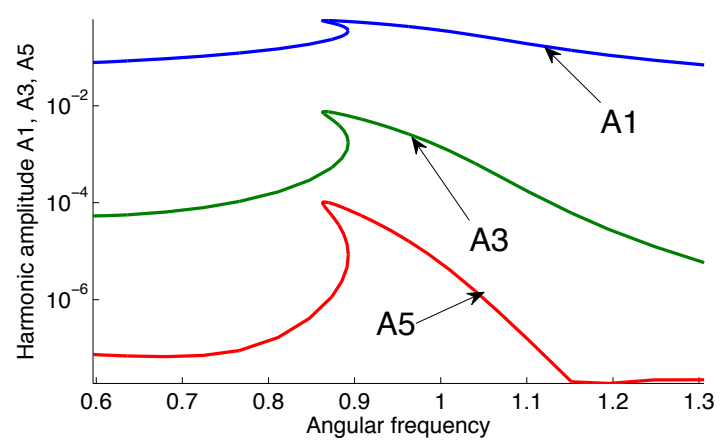

Figure 3: Duffing oscillator ( $\log$ scale), Eq. (1) with $\mu=0.05, \alpha=-0.5$ and $f=0.05$, "softening" system. Amplitude of harmonics 1, 3 and 5 versus forcing angular frequency $\lambda$.

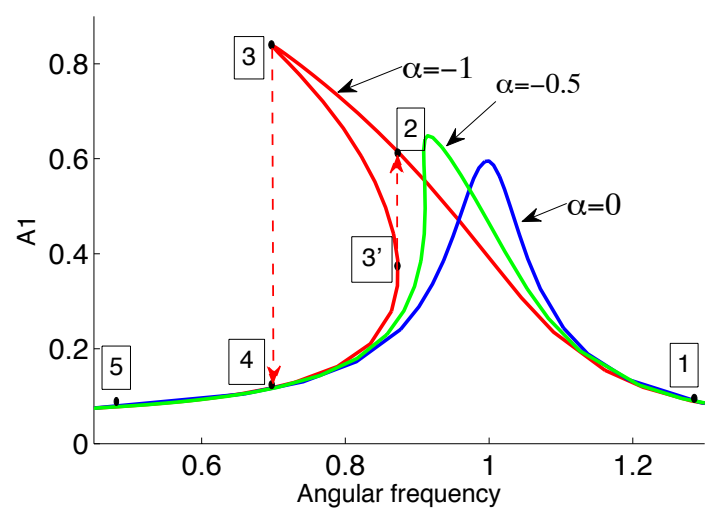

Figure 4: Duffing oscillator, Eq. (1) with $\mu=0.05$ and $f=0.04 . A 1$ amplitudes versus angular frequency when $\alpha=-1 ; \alpha=-0.5$ and $\alpha=0$. 
of the damping through the ratio $3 \alpha / 4 \mu^{2}$.

To sum up, the key idea of the global procedure is the quadratic recast. It provides a close form solution to the continuation problem in the frequency domain with an arbitrary high number of harmonics [24]. In addition, neither any iterative process nor any temporal sampling is required, thus avoiding low convergence and possible aliasing problems. The Duffing model introduced here is a pedagogical introduction to the HBM using the Manlab software. It is rather representative of the various theoretical models of free and encapsulated bubbles in viscous fluid that will now be analyzed.

\section{Bubble in viscous fluid: the "RPNNP" model.}

Let $R$ be the radius of the vibrating bubble, $R_{0}$ the radius at rest, the "RPNNP" equation is

$$
\rho_{L}\left[R \ddot{R}+\frac{3}{2} \dot{R}^{2}\right]=\left(P_{0}+\frac{2 \sigma}{R_{0}}\right)\left(\frac{R_{0}}{R}\right)^{3 \varsigma}-\frac{2 \sigma}{R}-4 \eta_{L} \frac{\dot{R}}{R}-P_{0}+P \cos \Omega t,
$$

where $P_{0}$ is the static pressure in the fluid, $\rho_{L}$ the fluid density, $\sigma$ the surface tension, $\eta_{L}$ the fluid viscosity, $\Omega$ the angular frequency of the plane pressurewave and $\varsigma$ the polytropic exponent.

In order to be able to recast this expression in the spirit of quadratic form, we consider isothermal oscillations: $\varsigma=1$. It is obvious that this working assumption has poor reality but responds to numerical limitations of the present Manlab ${ }^{\circledR}$ software: derivation of non-integer order are not yet implemented in it.

We note $\tau=\omega t$ and $\frac{d X}{d \tau}=\dot{X}$. By using $u=\frac{R}{R_{0}}, v=\frac{\dot{R}}{R_{0}}, x=\frac{1}{u}, y=x^{2}$, $z=v^{2}$ and $f=P \cos \Omega t$. Eq. (8) can be rewritten:

$\dot{v}=-\frac{P_{0}}{\rho_{L} \omega^{2} R_{0}^{2}} x-\frac{2 \sigma / R_{0}}{\rho_{L} \omega^{2} R_{0}^{2}} y+\frac{P_{0}+2 \sigma / R_{0}}{\rho_{L} \omega^{2} R_{0}^{2}} y^{2}-\frac{4 \eta_{L}}{\rho_{L} \omega R_{0}^{2}} y v-\frac{3}{2} x z+\frac{1}{\rho_{L} \omega^{2} R_{0}^{2}} x f$.

where the respective coefficients are:

$$
A=\frac{P_{0}}{\rho_{L} \omega^{2} R_{0}^{2}}, B=\frac{2 \sigma / R_{0}}{\rho_{L} \omega^{2} R_{0}^{2}}, C=\frac{4 \eta_{L}}{\rho_{L} \omega R_{0}^{2}} \text { and } D=\frac{1}{\rho_{L} \omega^{2} R_{0}^{2}},
$$

with $P_{0}=1,013.10^{5} \mathrm{~Pa}, \rho_{L}=10^{3} \mathrm{~kg} / \mathrm{m}^{3}, \sigma=72.10^{-3} \mathrm{~N} / \mathrm{m}, \eta_{L}=10^{-3}$ Pa.s, $\omega=10^{6} \mathrm{rad} / \mathrm{s}, R_{0}=2.10^{-6} \mathrm{~m}$ (air bubble in water of micrometer size studied within the megahertz range).

Equation (9) can recover the form of a quadratic system leading to the 
following state space extension of vector $Z$ :

$$
\left\{\begin{array}{ccccc}
\dot{u} & = & v & \\
\dot{v} & = & & -A x-B y & +(A+B) y^{2}-C y v-\frac{3}{2} x z+D x f \\
0 & = & 1 & y & -u x \\
0 & = & z & -x^{2} \\
0 & = & \underbrace{\underbrace{}_{m(\dot{Z})}}_{c(t)}= & \underbrace{f}_{l(Z)} & -v^{2}
\end{array}\right.
$$

where space vector is $Z=[u, v, x, y, z, f]$.

We observe that the compact expression of the equivalent quadratic system is analogous to (3). The unknown vector $Z$ (size $N_{e}=5+1$ ) contains the original components $u, v$, plus the auxiliary variables $x, y, z, f$ that have been added to transform (8) into the quadratic form (11).

Remember that vector $Z$ is written in the general form (4), thus

$$
u=\frac{R}{R_{0}}=u_{0}+\sum_{k=1}^{H}\left(a_{k} \cos k \Omega t+b_{k} \sin k \Omega t\right) .
$$

When the amplitude of the driving term is very weak $(P \simeq 0,01 P a)$ compared to the other terms of Eq.(8), the graph of amplitude $A_{1}$ versus angular frequency $\Omega$ describes the free response of the system. For low amplitudes, this amplitude/frequency curve remains tangential to the linear mode, $\Omega_{L}=\omega_{0}=8.7210^{6} \mathrm{rad} / \mathrm{s}$ (cf Table (1)), then gradually bends to the left with the activation intensity: this corresponds to a "softening" system as depicted in figures 3 and 4 .

Nota : In all the following figures, the angular frequency unit is $10^{6} \mathrm{rad} / \mathrm{s}$.

Changing radius $R_{0}$ of the bubble or considering the surface tension $(\sigma=$ $72.10^{-3} \mathrm{~N} / \mathrm{m}$ ) does not alter the global shape of the curve amplitude/angular frequency.

Only the "starting" angular frequency $\Omega_{L}$ for which the amplitude is non zero changes. This value is the undamped natural frequency of the linear system (nonlinear term set to zero) associated with Eq. (8): i.e. the Minnaert isothermal angular frequency when $\sigma=0$ or the Robinson[38] angular frequency when the surface tension is taken into consideration.

The angular frequencies obtained thanks to these two models are considered as functions of the radius and gathered in Table (1); these values fit very well with the results provided by Manlab software; we systematically find that $\Omega_{L}=$ $\omega_{\text {Minnaert }}$ (respectively $\Omega_{L}=\omega_{\text {Robinson }} ; \sigma \neq 0$ ) when $A_{1}=10^{-3}$. 


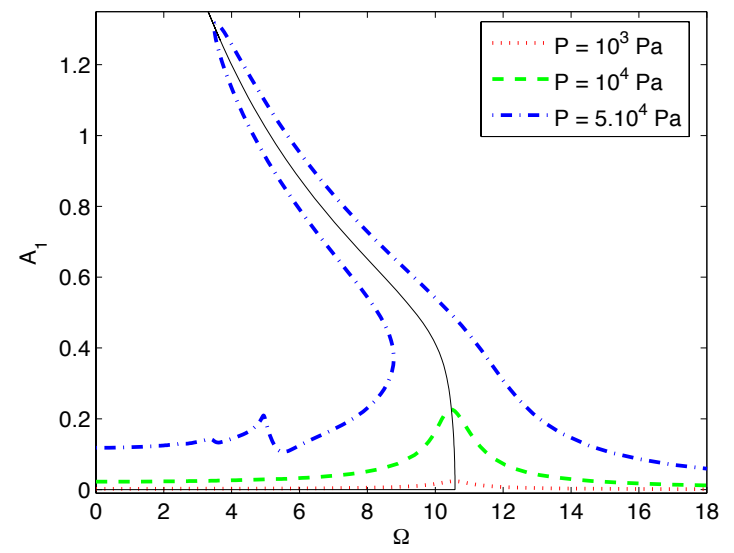

Figure 5: Frequency responses of the damped system (broken lines) and of the undamped system (continuous line)

The introduction of the fluid viscosity (for water, $\eta_{L}=10^{-3} \mathrm{Pa.s}$ ) in the modeling leads to a modification of the resonance behavior of the bubble. In order to observe this resonance, the damping term being non negligible in comparison with the other entities present in the equation, it is necessary to increase the amplitude $P$ of the driving oscillator. In fact we can observe (cf Fig. 5) the main resonance of the forced system which occurs when the driving harmonic component has an angular frequency close to that of the free system, in our case $\left(R_{0}=2 \mu \mathrm{m}, \omega_{0}=10.5710^{6} \mathrm{rad} / \mathrm{s}\right)$, it corresponds to the angular frequency derived by Houghton [40].

$$
\omega_{\text {Houghton }}=\frac{1}{R_{0}} \sqrt{\frac{3 \gamma\left(P_{0}+\frac{2 \sigma}{R_{0}}\right)-\frac{2 \sigma}{R_{0}}}{\rho_{L}}-\frac{4 \eta_{L}^{2}}{\rho_{L}^{2} R_{0}^{2}}} .
$$

Figure (6) depicts the evolution of the first, second and third harmonics versus angular frequency, the amplitude of the driving source being $P=5.10^{4} \mathrm{~Pa}$. The main resonance is given when $\Omega=\omega_{0}$, but there also exist secondary resonances, called super-harmonics, which occur when the angular activation frequency equals $\omega_{0} / 2$ or $\omega_{0} / 3$. At this secondary resonance, the amplitude of the second harmonic becomes higher than that of the first one (the fundamental). This has been found interesting [41] to transmit ultrasonic waves at the angular frequency $\Omega=\omega_{0} / 2$ with a sufficient amplitude $(P \geq 50 \mathrm{kPa}=0.5 \mathrm{~atm})$ in order to perform non linear (harmonic) imaging of the bubble in water or in tissues. However, when the amplitude of the driving wave is less than $10 \mathrm{kPa}=0.1 \mathrm{~atm}$, the non linear character of the bubble response is "erased" by the (water) viscous attenuation. Then, a linear damped oscillator model may be sufficient to characterize the bubble in water.

The resemblance of the frequency response curves with those obtained with the 


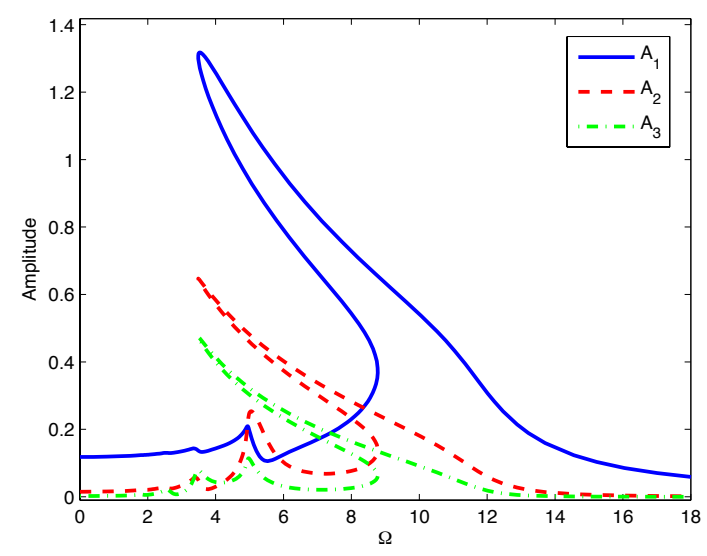

Figure 6: Amplitude of the first, second and third harmonics - $P=5.10^{4} \mathrm{~Pa}$

damped Duffing oscillator is obvious. This permits the use of the Duffing model as a "canonical" model of the system "free bubble in viscous fluid" and to characterize this system with the pair of parameters: the damping and the degree of nonlinearity.

Furthermore, considering an isolated bubble in a viscous fluid, a numerical experiment can be performed (using Matlab "ODE45" solver) in which the excitation amplitude is held constant but the frequency is varied continuously and linearly. Then, as described in the previous chapter, the jump phenomena in the amplitude response will be observed.

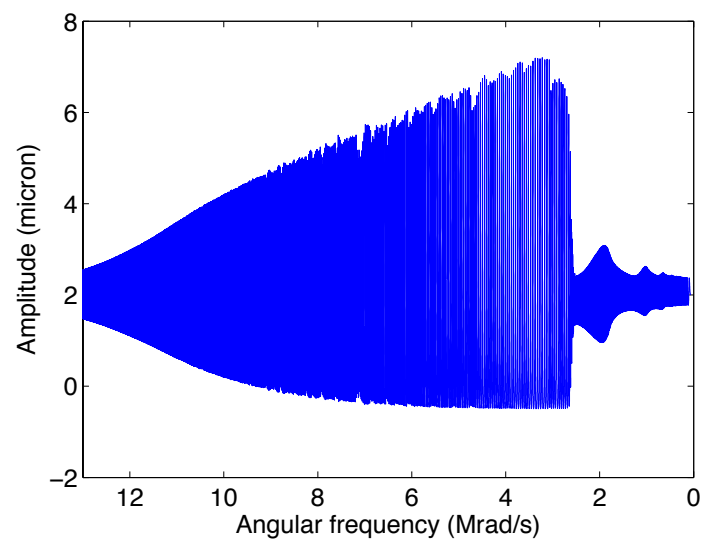

Figure 7: Temporal response of a 4-micron bubble to a linear down-chirp (range $[2.1,0] \mathrm{MHz}$ ) of constant amplitude $(50 \mathrm{kPa})$ 


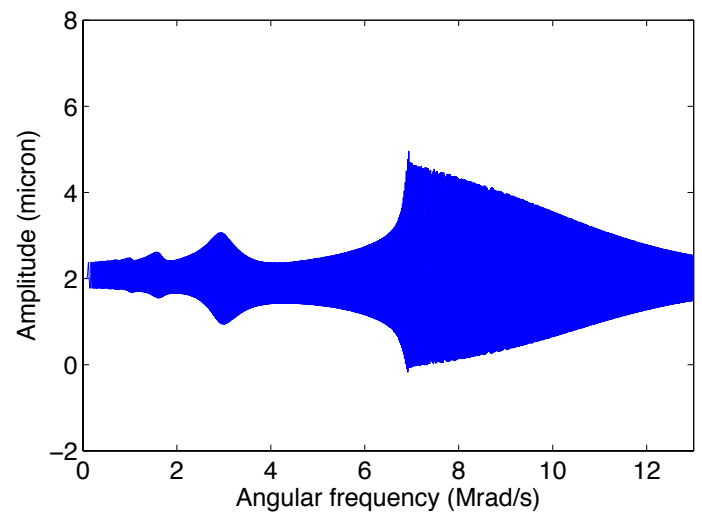

Figure 8: Temporal response of a 4-micron bubble to a linear up-chirp (range $[0,2.1] \mathrm{MHz}$ ) of constant amplitude $(50 \mathrm{kPa})$

If, for instance, the excitation is started at a frequency larger than the linear natural frequency, a decreasing frequency sweep will induce a larger jump in amplitude than an increasing frequency sweep starting at a frequency lower than the natural frequency (cf. figure 7 versus figure 8 ). In practice, this means that a higher signal to noise ratio will be obtained in the former case. In addition, this distinction between these increasing and decreasing sweeps can constitute the basis of a differential harmonic imaging that reveals the presence of the bubbles.

\section{Shelled contrast agent}

Most contrast agent bubbles are encapsulated in a shell that has two major effects on the acoustic response. First, the shell renders the bubble stiffer than a free gas bubble of equal size, making its resonance frequency higher than with the free bubble and reducing the non-linear effects. Secondly, the shell makes the bubble more viscous inducing an absorbed sound energy converted into heat instead of being radiated; the scatter/attenuation ratio is decreased. Numerous studies conclude that the influence of the shell is crucial and confirm that the properties of the acoustic field scattered by the agent are highly altered by the shell properties. Various shell models have been proposed; Fox and Hertzfeld [42] studied bubbles in sea water and underlined the influence of the organic shell, De Jong et al.[11, 12] introduced the empirical models of the Albunex, Church published a well-founded visco-elastic model [13] that assumes linear material properties but includes non-linear geometric effects. Angelsen and Hoff [14, 15] proposed an exponential stress strain relationship for the shell material. A few years ago, Marmottant introduced a "buckling" model [17] that accounts for buckling and rupture phenomena. These last two models will be analyzed in the following paragraphs. 


\subsection{Polymer-shell contrast Agent - Hoff model.}

Hoff considers contrast agents whose shell thickness, $e_{s}$, is low in comparison with internal and external radii, i.e. $e_{s}<<R_{1}, R_{2}$. This assumption enables one to reduce the equation of the movement. By noting $R_{2}$ the external radius of the shell and writing $R=R_{2}$, the equation reduces to:

$$
\begin{aligned}
\rho_{L}\left[R \ddot{R}+\frac{3}{2} \dot{R}^{2}\right] & =\left(P_{0}+\frac{2 \sigma}{R_{0}}\right)\left(\frac{R_{0}}{R}\right)^{3 \varsigma}-\frac{2 \sigma}{R}-4 \eta_{L} \frac{\dot{R}}{R} \\
-P_{0}+P \cos \Omega t & -12 \eta_{S} R_{0}^{2} e_{S} \frac{\dot{R}}{R^{4}}-12 \mu_{S} \frac{R_{0}^{2} e_{S}}{R^{3}}\left(1-\frac{R_{0}}{R}\right),
\end{aligned}
$$

where $\mu_{s}$ and $\eta_{s}$ are respectively the shear modulus and the shear viscosity of the shell that can be estimated via ultrasound attenuation measurements. Once again the behavior is assumed to be isothermal $(\varsigma=1)$ for present numerical possibilities of the Manlab software.

Quadratic form Recast: Equations (8) and (14) differentiate themselves only by the last two terms which sum up the shell contribution. In order to derive the associated quadratic form of Eq. (14), we re-use the variables defined in the former algebraic system (11), the latter being extended with two supplementary variables $\alpha=y^{2}$ and $\beta=\alpha x$ and two supplementary constants $E=\frac{12 \mu_{S} e_{S}}{\rho_{L} \omega^{2} R_{0}^{3}}$ and $F=\frac{12 \eta_{S} e_{S}}{\rho_{L} \omega R_{0}^{3}}$.

Then, Eq. (14) is equivalent to the following system of first order differential equations:

$$
\left\{\begin{array}{cccc}
u^{\prime} & = & v & \\
v^{\prime}= & & -A x-B y+(A+B-E) \alpha & -C v y-\frac{3}{2} x z+D x f-F \beta v \\
0= & 1 & y & -x u \\
0= & & & -x^{2} \\
0= & & +f & -v^{2} \\
0= & -P \cos \Omega t & \beta & -y^{2} \\
0= & & & -\alpha x \\
0= & & &
\end{array}\right.
$$

where $Z=[u, v, x, y, z, f, \alpha, \beta]^{t}$. Once again, the operators of the formal expression

$$
m\left(Z^{\prime}\right)=c(t)+l(Z)+q(Z, Z) .
$$

may be easily identified in the above system.

This model has been worked out by Hoff with the aim of characterizing a contrast agent with a gas core and a polymeric shell (such as AI-700 from Acusphere or Sonavist from Shering). On the assumption that the shell thickness equals $5 \%$ of the nominal radius $R_{0}$ of the contrast agent, the comparison with the theoretical prediction and the experimental measurements makes it possible to assess the viscoelastic coefficients of the shell[15]: $\mu_{s}=11.6 \mathrm{MPa}$ and $\eta_{s}=0.48$ Pa.s. 
Results: First, in order to evaluate the influence of the shear modulus $\mu_{s}$ of the UCA response, the shear viscosity is fixed to zero. Figure (9) shows the evolution of the fundamental amplitudes $A_{1}$ of four UCAs (radius $R_{0}=3 \mu \mathrm{m}$ ) of respective shear modulus values: 11.6, 50, 88 and $150 \mathrm{MPa}$ (these values are issued from [13]). To the increase of $\mu_{s}$ values corresponds an increase of the shell rigidity, this in turn limits the amplitude of the oscillations and fades the non-linear character of the UCA responses. The values of the angular frequencies for which the amplitudes start rising are in total agreement with those predicted by the close formulation derived from the linearization of Eq. (14)[15].

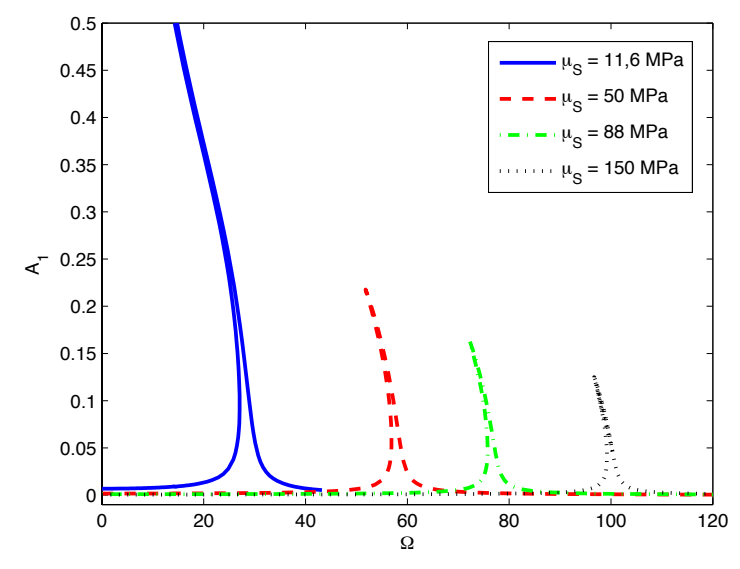

Figure 9: Influence of the shear modulus on amplitude $A_{1}$

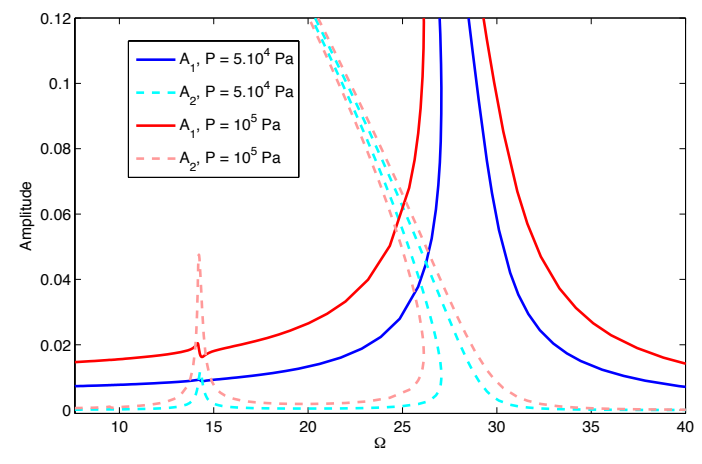

Figure 10: Amplitude of the first and second harmonics - zoom $\left(\mu_{s}=11.6 \mathrm{MPa}\right)$

The first and second harmonic amplitude values are illustrated in Fig. 10 for 
a fixed value of the shear modulus $\left(\mu_{s}=11.6 \mathrm{MPa}\right)$ and for two excitation levels $\left(P=5.10^{4} P a\right.$ i.e. $0.5 \mathrm{~atm}$ and $P=10^{5} \mathrm{~Pa}$ i.e. $\left.1 \mathrm{~atm}\right)$. The main resonance largely exceeds the secondary super-harmonic resonance. However, since in the latter case the secondary harmonic amplitude is greater than the fundamantal one when the amplitude of the driving source is large enough (typically, of the order of $100 \mathrm{kPa}$ ), the secondary resonance is still of interest for non-linear contrast imaging of tissues. Besides, for a given shear modulus (for instance $\mu_{s}=11.6 \mathrm{MPa}$ ), the viscosity of the shell leads to a strong reduction of the oscillation amplitudes as shown on graph 11. The resonance phenomenon is almost absent for viscosity values such as $\eta_{s}=0.48 P a . s$, which is in agreement with Hoff's conclusion. The experimental measurements[15] being performed on polydisperse UCA populations, the non-consideration of multiple scattering phenomena and the assumption that properties (thickness, visco-elasticity) are constant lead us to think that in this case, the shell viscosity assessment had certainly been over-estimated.

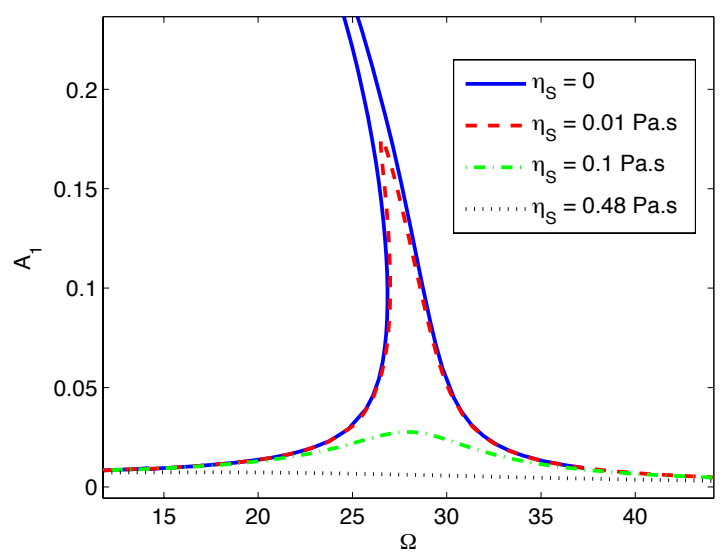

Figure 11: Influence of the viscosity of the shell on amplitude $A_{1}$

\subsection{Phospholipid-coated Contrast Agent: Marmottant model}

In this case, we are interested in the characterization of a lipid-shell UCA (e.g. Sonovue from Bracco; Imagent from Alliance)) whose model derived in [17] corresponds to the following Ordinary Differential Equation:

$\rho_{L}\left[R \ddot{R}+\frac{3}{2} \dot{R}^{2}\right]=\left(P_{0}+\frac{2 \sigma\left(R_{0}\right)}{R_{0}}\right)\left(\frac{R_{0}}{R}\right)^{3 \varsigma}-\frac{2 \sigma(R)}{R}-4 \eta_{L} \frac{\dot{R}}{R}-4 \kappa_{S} \frac{\dot{R}}{R^{2}}-P_{0}+P \cos \Omega t$. 
We limit ourselves to the elastic behavior of the shell interface (excluding buckling and rupture) for which the surface tension verifies

$$
\sigma(R)=\chi_{S}\left(\frac{R^{2}}{R_{b}^{2}}-1\right) .
$$

where $R_{b}$ stands for the threshold radius of the shell under which buckling of the phospholipid molecules occurs.

Quadratic form Recast: Let us note $g=x y, Z=[u, v, x, y, z, f, g]^{t}$ (variables $u, v, x, y, z$ and $f$ remain unchanged) and let us define the following constants:

$$
\begin{gathered}
A_{M}=\frac{2 \chi_{S}}{\rho_{L} \omega^{2} R_{0} R_{b}^{2}}, B_{M}=\frac{P_{0}}{\rho_{L} \omega^{2} R_{0}^{2}}, C_{M}=\frac{2 \chi_{S}}{\rho_{L} \omega^{2} R_{0}^{3}}, D_{M}=\frac{R_{0}^{2}}{R_{b}^{2}}-1, \\
E_{M}=\frac{4 \eta_{L}}{\rho_{L} \omega R_{0}^{2}}, \quad F_{M}=\frac{4 \kappa_{S}}{\rho_{L} \omega R_{0}^{3}}, G_{M}=\frac{1}{\rho_{L} \omega^{2} R_{0}^{2}} .
\end{gathered}
$$

Equation (17) can be rewritten in the quadratic form:

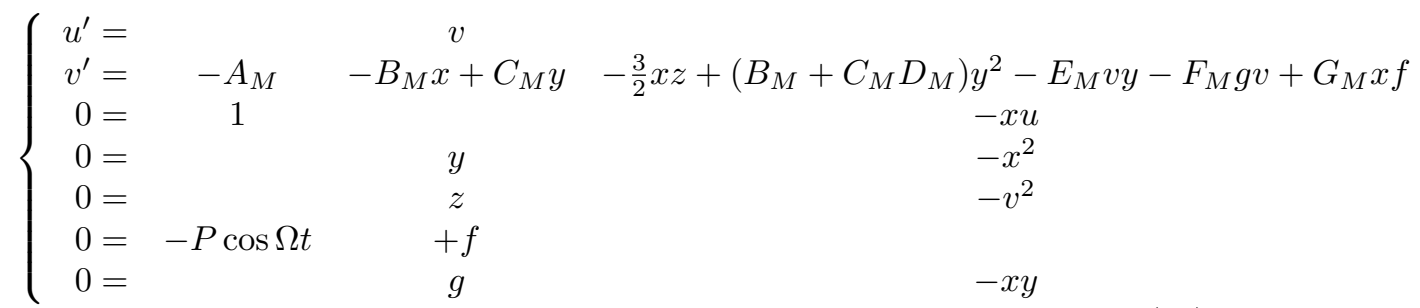

Figure 12 shows the natural $\left(P=10^{-2} P a\right)$ response of the undamped system $\left(R_{0}=R_{f}=1.98 \mu \mathrm{m}, \chi_{S}=0.55 \mathrm{~N} / \mathrm{m}\right.$ and $\left.\eta_{L}=\kappa_{S}=0\right)$ in addition to the forced response $\left(P=5.10^{4} \mathrm{~Pa}\right)$ of the damped system when only the liquid viscosity is taken into account $\left(\eta_{L}=10^{3} \mathrm{Pa.s}\right)$. Finally, the shell viscosity is also considered $\left(\kappa_{S}=7 \cdot 2 \cdot 10^{-9} N\right)$. The values of the parameters originate from [17] and [19].

Figure 12 shows that the system is hardening at the lower amplitudes of the activation source, then as the driving power increases, it softens. However, once again, taking into account the viscosity of both fluid and shell reduces drastically the oscillation amplitude and "erases" the non-linear character of the behavior. This result is in agreement with the work of Van der Meer and al [19] who demonstrate that a simple oscillator model derived by linearization of the Marmottant model is sufficient for describing the radial oscillations of an UCA driven by a $40 \mathrm{kPa}$ excitation amplitude. These conclusions were deduced from the experimental observations obtained using the Brandaris ultrafast video camera[19].

Figure 13 shows the evolution of the first and second harmonics as functions of angular frequency $\Omega$ for various amplitude levels $P$ of the driving source. One can easily isolate (on $A_{2}$ curve) resonances at $\Omega=\Omega_{L} / 2$ and at $\Omega=\Omega_{L} / 3$ when the source amplitude is greater than $100 \mathrm{kPa}$. It is interesting to note that 


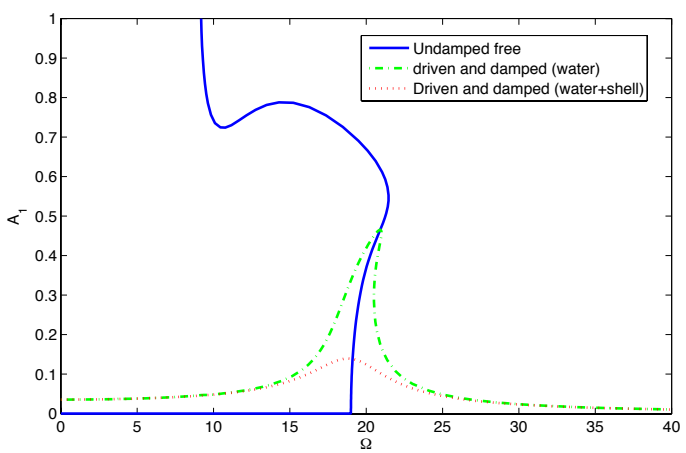

Figure 12: Marmottant model : Influence of the liquid and shell viscosities on amplitude $A_{1}$

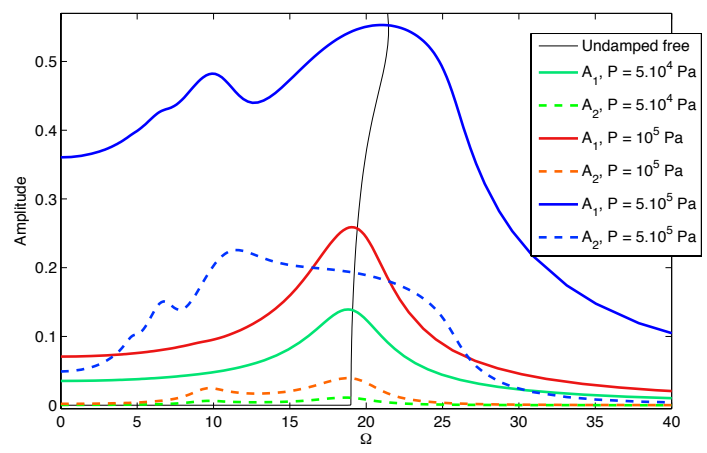

Figure 13: Influence of the excitation pressure on the first and second harmonics

for the two previous models (for similar values of $P$ ), at the secondary superharmonic resonance $\left(\Omega=\Omega_{L} / 2\right)$, the amplitude of the second harmonic is less than that of the fundamental. However, here, at the super-harmonic resonance and for the highest pressure level, the amplitude of the second harmonic exceeds its main resonance value; this situation has never been met before.

\section{Application to "small" bubble sizing}

The problem of detecting and sizing microbubbles in their host environment has received particular attention over the last thirty years. Concerning reflection measurements only, most techniques are based on the use of a "pumping" field $[1,2,3,4]$ whose frequency is close to the resonance frequency of the bubble to be detected, associated with an "imaging" field of a higher frequency. When interacting with the bubble and because of the latter's non-linear behavior, 
the two waves generate among all the sideband components sum and difference frequencies that exhibit unambiguous spectral peaks as functions of the bubble radius. This double frequency method is robust and is mainly implemented in two ways depending on the possible synchronization of the pump and imaging fields. Implementations that do not consider temporal dependence between these two fields were first introduced. Then, an improved version providing both lateral and axial resolutions was obtained with a pulsed Doppler approach [2]. Recently, a low frequency "manipulation" pulse modifying the scattering properties of the bubble and a high frequency imaging pulse, phase-controlled with relation to this manipulation wave, has made it possible to generate bubble detection signals[43]. However, when small bubbles are sought for (diameter comprised between $[1,10]$ microns), e. g. in relation to prevent decompression sickness and to obtain accurate assessment of the bubble distribution when using current medical ultrasounds (frequency range $[1,15] \mathrm{MHz}$ ), the double frequency method becomes impracticable. Indeed, for these bubble sizes, the low frequency required varies from $0.65 \mathrm{MHz}$ up to $6.5 \mathrm{MHz}$ and enters the frequency range of the imaging field. Consequently, the relevant sideband components fall out of the bandpass of the imaging transducers.

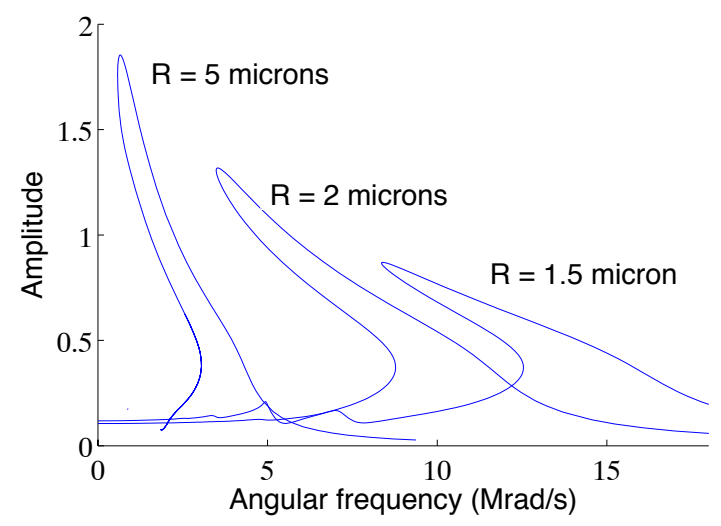

Figure 14: Frequency responses of bubbles (diameters 10, 4, 3 microns) to a pressure wave of $50 \mathrm{kPa}$

We propose here to use the non-linear frequency bubble responses (same conditions as in paragraph 3) in order to obtain a small microbubble detection/sizing tool. Figures 14 and 15 that depict the frequency responses of air bubbles in water(same physical parameters as in $\$ 3$ ) with size comprised between 1 and 10 microns reveal that bubbles of diameter larger than 2 microns exhibit strong non-linearities when excited by an acoustical pressure wave of $50 \mathrm{kPa}$. But this is not the case for bubbles whose radii are inferior to one micron. However, figure (16) shows that doubling the pressure amplitude of a 2 -micron in diameter bubble to $100 \mathrm{kPa}$ restitutes the non-linear character of its frequency response. 


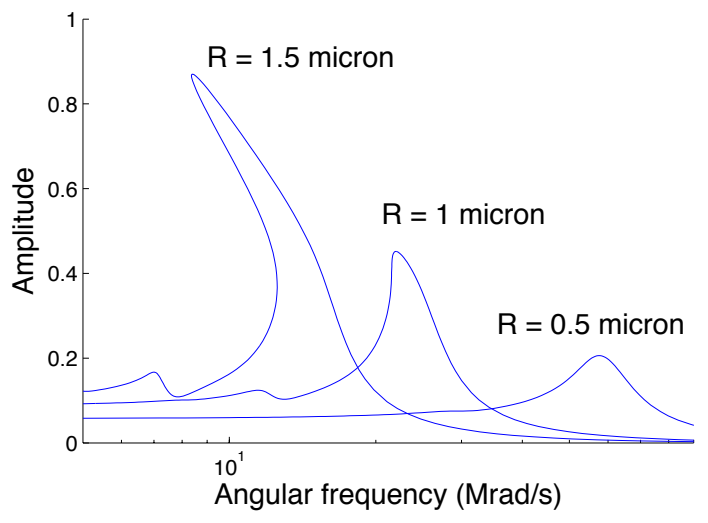

Figure 15: Frequency responses (log scale) of bubbles (diameters 3, 2, 1 microns) to a pressure wave of $50 \mathrm{kPa}$

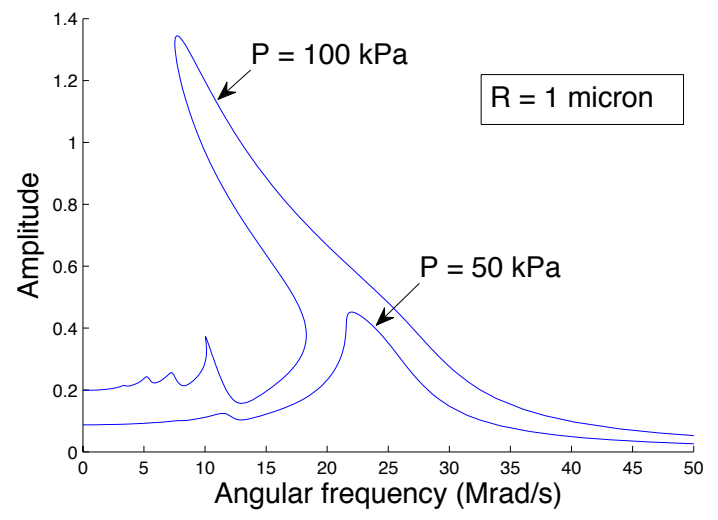

Figure 16: Frequency responses of 2-micron in diameter bubble to pressure waves of $50 \mathrm{kPa}$ ("linear" behavior) and $100 \mathrm{kPa}$ (nonlinear behavior)

The non-linear behavior induces the presence of jump-up and jump-down frequencies which are relevant characteristics of the size of the bubbles placed in a given viscous fluid. Then, if a bubble of a given size is insonified with linearly modulated chirps of decreasing and rising frequency sweeps whose limits encompass its jump-up frequency, the response of the bubble will be maximal when compared with those for which the jump-up frequency is not included in this interval. For instance, the amplitude signal backscattered by a 4-micron bubble is 3 or 4 greater compared with the signals generated by bubbles whose radius differs only by 1 micron. This resonance phenomenon is illustrated in the four following figures (fig. 17-20). In these numerical experiments with various bubble sizes dedicated to the detection of 4-micron bubbles, the activation signal 
of $530 \mathrm{~ms}$ duration is linearly modulated from $1.55 \mathrm{MHz}(9.7 \mathrm{Mrad} / \mathrm{s})$ down to $1.25 \mathrm{MHz}(7.8 \mathrm{Mrad} / \mathrm{s})$ during the first half of the duration, then up-modulated to $1.55 \mathrm{MHz}$ during the second half. The temporal responses of the bubble are obtained using "ODE45" Matlab solver.

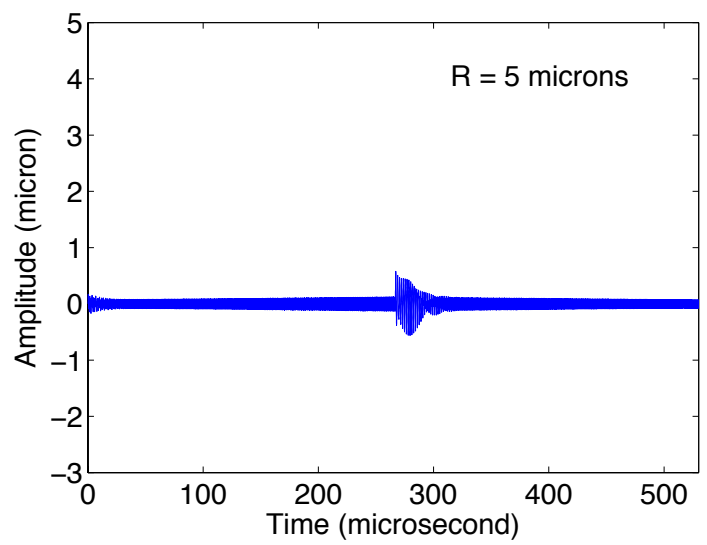

Figure 17: Response of a 10-micron bubble to a linear down/up chirp (range [1.25-1.55 MHz]) 


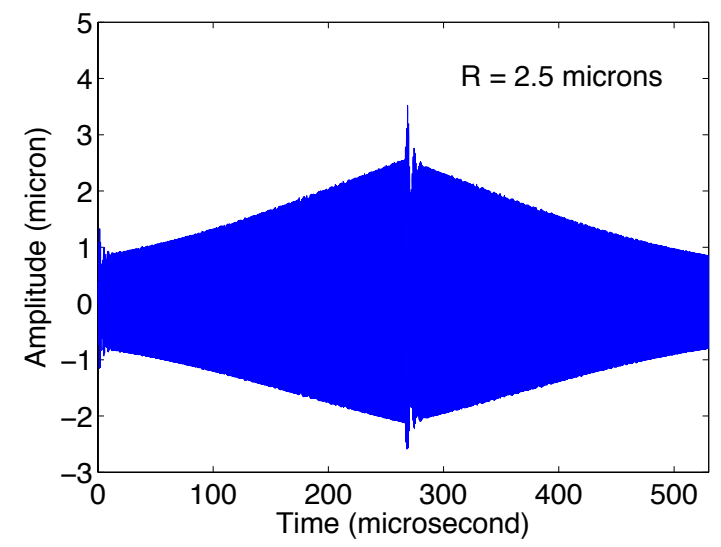

Figure 18: Response of a 5-micron bubble to the same pressure wave as in fig. 17

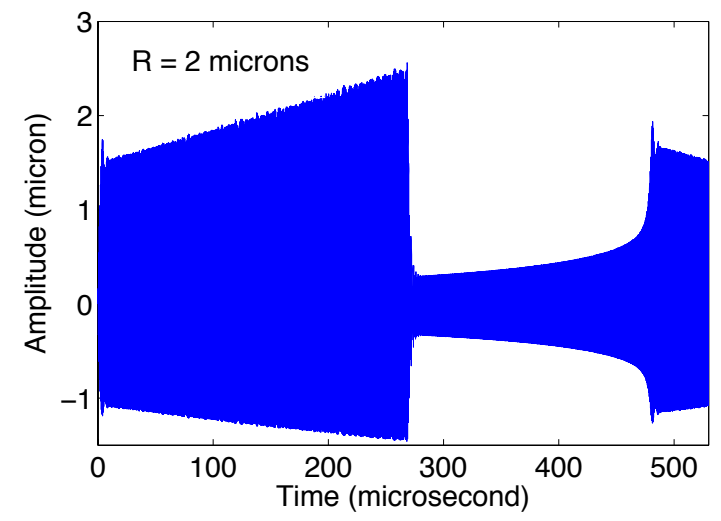

Figure 19: Response of a 4-micron bubble to the same pressure wave as in fig. 17

One can observe that the temporal responses of the bubbles whose diameters are different by more than 1 micron compared to the 4-micron reference bubble present symmetrical shapes. This results from the fact that, for these bubbles, the jump-up frequency is not crossed over by the frequency sweep. Therefore, only either the upper branch or the lower branch of their frequency response is followed in a reversible way. On the contrary, due to the jump, the 4-micron bubble reveals a hysteretic (asymmetrical) behavior. Hence, the idea of extracting the signal envelope and of calculating the differences between the samples recorded at the same instantaneous frequency which leads to a nonlinear signature of each bubble.

Figure 21 shows these signatures and proves, for instance by applying a 


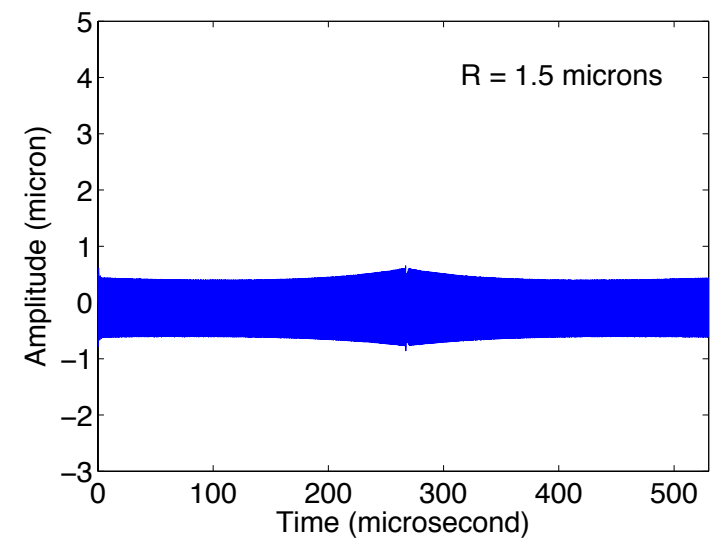

Figure 20: Response of a 3-micron bubble to the same pressure wave as in fig. 17

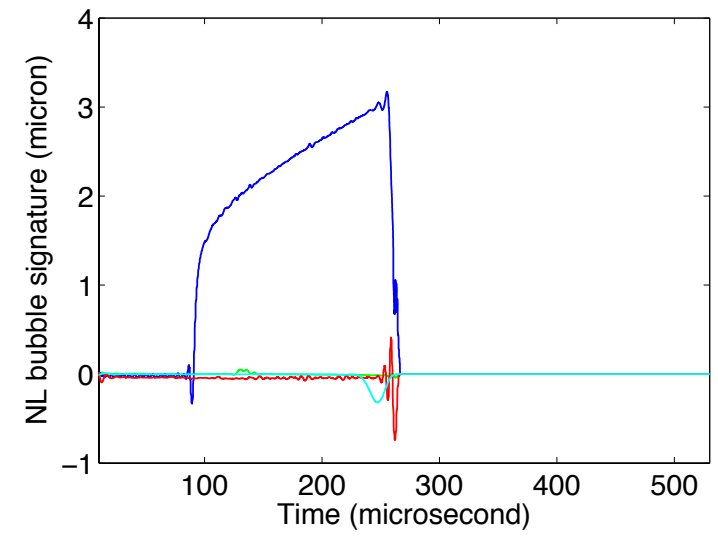

Figure 21: Nonlinear signatures of various bubbles in case of an activation for a 4-micron bubble detection

simple threshold, that it is possible to detect and discriminate bubbles whose diameters are in the $[1,10]$ micron range with a theoretical resolution inferior to 1 micron. 


\section{Conclusion}

The characterization of bare or coated (contrast agent) microbubbles is of great interest in many industrial and medical fields of application. The search for relevant parameters (precursors) confirming the presence of these microbubbles, or leading to their size distribution, or providing an unambiguous assessment of their navigation conditions (free evolution versus endothelium cell or wall surface adhesion) requires more in depth research into nonlinear tools and methodologies. In order to extend the standard domain of linear analysis, this paper demonstrates that the high order harmonic balance method (HBM) is a good procedure since it provides natural means, implemented in the Manlab ${ }^{\circledR}$ suite (open-access software), for investigating the behavior of such a nonlinear system (i.e. the microbubble in water). For this purpose, a specific methodology has been designed which consists in examining the NonLinear Ordinary Differential Equation (NL-ODE) that governs the time evolution of the bubble radius in response to ultrasonic activation. A local periodic solution has been devised whose "tracking" is facilitated by the use of a specific continuation technique that operates in conjunction with the HBM. The "manual" pre-processing of the NL-ODE consists in a quadratic recast of the nonlinear equations. In the frequency domain, this provides a closed-form solution which possesses an arbitrary number of harmonics to the continuation problem. Following this methodology, various theoretical models of bubble response in viscous fluid have been studied : the "RNNP" model depicts a free microbubble response; the Hoff and Marmottant models are dedicated to shelled contrast agents with polymeric and phospholipid shells respectively.

For a start, the coherence of the results obtained thanks to the global procedure has been validated, firstly by demonstrating that if the number of harmonics considered in the calculus is not to be underestimated, the rapid asymptotical convergence observed warrants that an arbitrary precision can be reached. Secondly, the parametrical study that has been performed agrees with the results reported in the literature especially as far as the primary and secondary resonances are concerned. Moreover, jump-up and jump-down frequencies are revealed and quantified and the shape of the frequency response reveals the straightforward analogy with a softening or hardening damped Duffing oscillator according to the strain law of the shell material. In addition, the HBM can be used as a predictive tool that, for instance, makes it possible to anticipate the hardening or softening behavior of the (encapsulated) microbubble in its host fluid, to distinguish a linear from a nonlinear regime of the bubble response according to the amplitude of the forcing, or to assess the damping attenuation which affects each harmonic component according to the viscous properties of the host medium and of the shell material. This information is crucial for the production of anti-cavitation fluid, the monitoring of the void fraction in nuclear coolant (liquid sodium) or the design of medical ultrasound contrast agents. Lastly, the analysis of the bubble frequency responses shows that an ultrasound spectroscopic method can be designed which considers the jumpup frequency as an unambiguous parameter function of the bubble diameter. 
Using down/up chirps whose frequency ranges contain the jump-up frequency associated with a given diameter improves the detection signal to noise ratio of that bubble. Indeed, the sizing of $[1,10]$-microns bubbles is not possible by using the so-called "double frequency" techniques since the sideband components generated fall out of the bandpass of the medical ultrasound transducers. Theoretically, the present methodology offers a resolution in the detection inferior to 1 micron for a bubble of 4 microns. 
[1] V.L. Newhouse, P.M. Shankar, "Bubble size measurements using the nonlinear mixing of two frequencies", J. Acoust. Soc. Am. 75, 1473-1477 (1984)

[2] D. Cathignol, J. Y. Chapelon, V.L. Newhouse and P.M. Shankar, "Bubble sizing with high spatial resolution", IEEE Tans. Ultrason. Ferro. Freq. Control, 37 (1), 30-37 (1990)

[3] C. X. Deng, F. L. Lizzi, A. Kalisz, A. Rosado, R. H. Silverman, and D. J. Soleman, "Study of ultrasonic agents using a dual-frequency band echnique", Ultrason. Med. Biol., 26 (5), 819-831, (2000)

[4] C. Y. Wu and J. Tsao, "The ultrasonic weak short-pulse response of microbubbles based on a two-frequency approximation", J. Acoust. Soc. Am. 114 (5), 2662-2671 (2003)

[5] Yu. N. Savchenko, Yu. D. Vlasenko, V. N. Semenenko, "Experimental investigations of high speed supercavitating flows", J. Hydromechanics (72), 103-102 (1998)

[6] Y. L. Young and S. A. Kinnas, "Analysis of supercavitating and surfacepiercing propeller flows via BEM", Computational Mechanics 32, $269 ? 280$ (2003)

[7] K. S. Suslick, S. E. Skrabalak, "Sonocatalysis" In Handbook of Heterogeneous Catalysis, vol. 4; G. Ertl, H. Knzinger, F. Schuth, J. Weitkamp Eds.; Wiley-VCH: Weinheim, Germany, 2006-2017 (2008)

[8] R. D. Watkins, L. M. Barrett and J. A. McKnight, "Ultrasonic waveguide for use in the sodium coolant of fast reactors", Nuclear Energy, 27, 85-89, (1988)

[9] B. H. Kim, V. S. Yughay, S. T. Huang, B. H. Kim, J. H. Park, C. S. Choi, "Hydrogen bubble characteristics during a water-sodium leak accident in a stream generator", J. Industrial Engineering Chemistry, 6 (6), 395-402, (2000)

[10] J. C. Buckey, D. A. Knaus, D. L. Alvarenga, M. A. Kenton, P. J. Magari, "Dual-frequency ultrasound for detecting and sizing bubbles", Acta Astronica, 56 1041-1047, (2005)

[11] N. de Jong, L. Hoff, T. Skotland and N. Bom, "Absorption and scatterer of encapsulated gas filled microspheres: theoretical considerations and some measurements", Ultrasonics, 30, 95-103 (1992)

[12] N. de Jong, L. Hoff, "Ultrasound scattering properties of Albunex microspheres", Ultrasonics, 31, 175-181, (1993)

[13] C. C. Church, "The effects of an elastic solid surface layer on the radial pulsations of gas bubbles" , J. Acoust. Soc. Am. 97, 1510-1521 (1995) 
[14] B. A. Angelsen, L. Hoff, and T.F. Johansen, "Simulation of gas bubble scattering for large Mach-number, IEEE Ultrasonics symposium Proceedings, 505-508, (2000)

[15] L. Hoff, , P.C. Sontum, J.M. Hovem, "Oscillations of polymeric microbubbles: effect of the encapsulating shell", J. Acoust. Soc. Am. 107 (4), 2272-2280 (2000)

[16] X. Yang and C. C. Church , "A model for the dynamics of gas bubbles in soft tissue", J. Acoust. Soc. Am. 118, 3595-3606 (2005)

[17] P. Marmottant, S. Van der Meer, M. Emmer, M. Verluis, N. de Jong, S. Hilgenfeldt, D. Lohse, "A model for large amplitude oscillations of coated bubbles accounting for buckling and rupture", J. Acoust. Soc. Am. 118 (6), 3499-3505 (2005)

[18] K. Tsiglifis and N. A. Pelekasis, "Nonlinear radial oscillations of encapsulated microbubbles subject to ultrasound: The effect of membrane constitutive law", J. Acoust. Soc. Am. 123, 4059-4070 (2008)

[19] S. M. van der Meer, B. Dollet, M. M. Voormolen, C. T. Chin, A. Bouakaz, N. de Jong, M. Versluis, and D. Lohse, "Microbubble spectroscopy of ultrasound contrast agents" , J. Acoust. Soc. Am. 121, 648-656 (2007)

[20] A. A. Doinikov, J. F. Haac and P. A. Dayton, "Resonance frequencies of lipid-shelled microbubbles in the regime of nonlinear oscillations", Ultrasonics 49 (2), 263-268, (2009)

[21] P. D. Krishna, P. M. Shankar, V. L. Newhouse "Subharmonic generation from ultrasonic contrast agents," Phys Med Biol. 44, 681?694, (1999).

[22] P. M. Shankar, P. D. Krishna, V. L. Newhouse "Subharmonic backscattering from ultrasound contrast agent", J. Acoust. Soc. Am. 106(4), 2104-2110, (1999)

[23] http://manlab.lma.cnrs-mrs.fr/ Manlab is a Matlab package for interactive continuation and bifurcation analysis of non linear systems of equations (date last viewed 4/15/09)

[24] B. Cochelin, C. Vergez, "A high order purely frequential harmonic balance formulation for continuation of periodic solutions", Journal of Sound and Vibration, 324, 243-262 (2009)

[25] W. Lauterborn, "Numerical investigation of nonlinear oscillations of gas bubbles in liquids", J. Acoust. Soc. Am. 59, 283-293 (1976)

[26] J. B. Keller and M. Miksis, "Bubble oscillations of large amplitude", J. Acoust. Soc. Am. 68, 628-633 (1980)

[27] A. H. Nayfeh, W.S. Saric, "Non-linear acoustic response of a spherical bubble", Journal of Sound and Vibration, 30 (4), 445-453 (1973) 
[28] A. H. Nayfeh, "Perturbation Methods", John Wiley \& Sons Inc. NY(1973)

[29] A. H. Nayfeh, D. T. Mook, "Nonlinear oscillations", John Wiley \& Sons Inc. NY(1995), Wiley-VCH: Weinheim, Germany (2004)

[30] A. Francescutto and R. Nabergoj, "Steady-state oscillations of gas bubbles in liquids: Explicit formulas for frequency response curves", J. Acoust. Soc. Am. 73, 457-460 (1983)

[31] J. S. Allen and R. A. Roy, "Dynamics of gas bubbles in viscoelastic fluids. I. Linear viscoelasticity", J. Acoust. Soc. Am. 107, 3167-3178 (2000)

[32] A. Prosperetti, "Nonlinear oscillations of gas bubbles in liquids: steadystate solutions" , J. Acoust. Soc. Am. 56, 878-885 (1974)

[33] D. B. Khismatullin, A. Nadim, "Radial oscillations of encapsulated microbubbles in viscoelastic liquids", Physics of Fluids 14 (10), 3534-3557 (2002)

[34] A. Shima, T. Tsujino, H. Nanjo, "Nonlinear oscillations of gas bubbles in viscoelastic fluids", Ultrasonics 24 (3), 142-147 (1986)

[35] O. M. Mukdadi, H.-B. Kim, J. Hertzberg and R. Shandas, "Numerical modeling of microbubble backscatter to optimize ultrasound particle image velocimetry imaging: initial studies", Ultrasonics 42 (10), 1111-1121 (2004)

[36] B. Cochelin, "A path-following technique via an asymptotic numerical method ", Computers and Structures 53 (5), 1182-1192 (1994)

[37] M.J. Brennan, I. Kovacic, A. Carrella, T.P. Waters, "On the jump-up and jump-down frequencies of the Duffing oscillator", Journal of Sound and Vibration, 318, 1250-1261 (2008)

[38] R. B. Robinson, R. H. Buchanan, "Undamped free pulsations of an ideal bubble", Proceedings of the Physical Society, Section B, 69(9), 893-900 (1956)

[39] L. Liu, J.P. Thomas, E.H. Dowell, P. Attar, K.C. Hall, "A comparison of classical and high dimensional harmonic balance approaches for a Duffing oscillator", Journal of Computational Physics 215, 298-320 (2006)

[40] G. Houghton, "Theory of bubble pulsation and cavitation", Journal of the Acoustical Society of America, 35 (9), 1387-1393, (1963)

[41] A. Bouakaz, N. de Jong, C. Cachard, "Standard properties of ultrasound contrast agents, Ultrasound in Medicine and Biology", 24 (3), 469-472 (1998).

[42] F.E. Fox and K.F. Hertzfield, "Gas bubbles with organic skin as cavitation nuclei", J. Acoust. Soc. Am. 26, 984-989, (1954)

[43] S.E. Masoy, O. Standal, P. Nasholm, J. Johansen, B. Angelsen "SURF Imaging : In Vivo Demonstration of an Ultrasound Contrast Agent Detection Technique" IEEE UFFC, 55 (5), 1112-1121, (2008) 


\section{TABLE CAPTIONS}

\begin{tabular}{ccccccc} 
Radius $R_{0}(\mu \mathrm{m})$ & 1 & 2 & 3 & 5 & 10 & 17 \\
\hline \hline$\omega_{\text {Minnaert }}\left(10^{6} \mathrm{rad} / \mathrm{s}\right)$ & 17.43 & 8.72 & 5.81 & 3.49 & 1.74 & 1.025 \\
$\omega_{\text {Robinson }}\left(10^{6} \mathrm{rad} / \mathrm{s}\right)$ & 24.33 & 10.58 & 6.66 & 3.80 & 1.82 & 1.05
\end{tabular}

Table 1: Linear angular frequency without (Minnaert) and with (Robinson) consideration of the surface tension 


\section{FIGURE CAPTIONS}

Fig. 1. Duffing oscillator, Eq. (1) with $\mu=0.05, f=1.0$ and $\alpha=1$, "hardening" system, as in ([39]). The figure shows the amplitude of harmonics 1, 3 and 5 versus forcing angular frequency $\lambda$. Continuous line : results when 5 harmonics are included ( $\mathrm{H}=5$ in Eq. (4)). Dotted line : results when 9 harmonics are included.

Fig. 2. Duffing oscillator, same conditions as in Fig. (1): zoom of the region $\omega<0.6$.

Fig. 3. Duffing oscillator (log scale), Eq. (1) with $\mu=0.05, \alpha=-0.5$ and $f=0.05$, "softening" system. Amplitude of harmonics 1, 3 and 5 versus forcing angular frequency $\lambda$.

Fig.4. Duffing oscillator, Eq. (1) with $\mu=0.05$ and $f=0.04$. $A 1$ amplitudes versus angular frequency when $\alpha=-1 ; \alpha=-0.5$ and $\alpha=0$.

Fig. 5. Frequency responses of the damped system (broken lines) and of the undamped system (continuous line)

Fig. 6. Amplitude of the first, second and third harmonics - $P=5.10^{4} P a$

Fig. 7. Temporal response of a 4-micron bubble to a down-modulated signal (range $[2.1,0] \mathrm{MHz}$ ) of constant amplitude (50 kPa) Fig. 8. Temporal response of a 4-micron bubble to a up-modulated signal (range $[0,2.1] \mathrm{MHz}$ ) of constant amplitude (50 kPa) Fig. 9. Influence of the shear modulus on amplitude $A_{1}$

Fig. 10. Amplitude of the first and second harmonics - zoom $\left(\mu_{s}=11.6 \mathrm{MPa}\right)$

Fig. 11. Influence of the viscosity of the shell on amplitude $A_{1}$

Fig. 12. Marmottant model : Influence of the liquid and shell viscosities on amplitude $A_{1}$

Fig. 13. Influence of the excitation pressure on the first and second harmonics

Fig. 14. Frequency responses of bubbles (diameters 10, 4, 3 microns) to a pressure wave of $50 \mathrm{kPa}$

Fig. 15. Frequency responses (log scale) of bubbles (diameters $3,2,1$ microns) to a pressure wave of $50 \mathrm{kPa}$

Fig. 16. Frequency responses of 2-micron bubble to pressure waves of $50 \mathrm{kPa}$ ("linear" behavior) and $100 \mathrm{kPa}$ (nonlinear behavior)

Fig. 17. Response of a 10-micron bubble to a decrease and increase in linear modulation (range [1.25-1.55 MHz])

Fig. 18. Response of a 5 -micron bubble to the same pressure wave as in fig. 17

Fig. 19. Response of a 4 -micron bubble to the same pressure wave as in fig. 17

Fig. 20. Response of a 3-micron bubble to the same pressure wave as in fig.

Fig. 21. Nonlinear signatures of various bubbles in case of an activation for a 4-micron bubble detection 